\title{
HLA haplotype sharing by siblings with rheumatoid arthritis: evidence for genetic heterogeneity
}

\author{
DAVID M GRENNAN, PAUL A SANDERS, PHILIP A DYER, AND \\ RODNEY HARRIS
}

From the University of Manchester Rheumatic Diseases Centre, Hope Hospital, Salford M6 8HD; and the University Department of Medical Genetics, St Mary's Hospital, Manchester

SUMMARY HLA haplotype sharing was studied in 35 sibships in which there were two or more $\frac{0}{2}$ members with rheumatoid arthritis (RA). Haplotype sharing by RA siblings was random in 15 oे

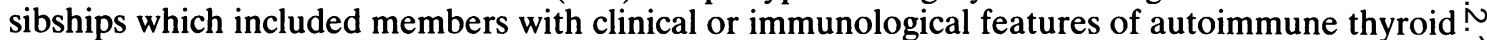
disease. In the remaining 20 'non-thyroid' sibships the frequencies of RA siblings sharing 0,1 , or $\vec{N}$ 2 haplotypes were $0.04,0.48$, and 0.48 respectively $(p=0.006) .67 \%$ of RA probands in the 을 'thyroid' families and $90 \%$ in the other families were HLA-DR4 positive. It is suggested that $\rightarrow$ there is genetic heterogeneity in the pathogenesis of RA with at least two independent genes 7 within the major histocompatibility complex (MHC) predisposing to RA. One gene is in linkage $\frac{0}{2}$ disequilibrium with HLA-DR4, while results of comparison of DR antigen frequencies in DR4 ฏ negative RA and control groups suggest that the other is in linkage disequilibrium with HLADR1 and 3. In the thyroid disease families both genes are frequently present and as either mæ predispose to arthritis, HLA haplotype sharing is random. The frequencies of HLA haplotype sharing in the 'non-thyroid' families suggest that there is a dominant susceptibility gene in linkage disequilibrium with HLA-DR4, whose frequency is $5 \%$ and penetrance about $20 \%$.

Key words: autoimmune thyroid disease, gene frequency, dominant gene.

Rheumatoid arthritis (RA) is thought to be caused by an interaction between a polygenic disease susceptibility and unknown environmental factors. ${ }^{1}$ There is a well documented association between RA and HLA-DR4 in most populations studied, ${ }^{2}$ suggesting that a gene or genes within the MHC may be responsible for at least part of this genetic susceptibility. The presence of DR4 negative RA patients could represent linkage disequilibrium between DR4 and a single RA susceptibility gene within the MHC or genetic heterogeneity with different susceptibility genes within the MHC for DR4 positive and negative RA. We have suggested previously that genes for autoimmune thyroid disease may predispose to RA independently of DR4. ${ }^{3}$ In the present study we have compared HLA haplotype sharing by DR4 positive and negative RA probands with their affected sibs in sibships which either do or do not have individuals with autoimmune thyroid

Accepted for publication 24 July 1985.

Correspondence to Dr D M Grennan, University of Manchester Rheumatic Diseases Centre, Hope Hospital, Salford M6 8HD. disease. If there is a single RA susceptibility gene within the MHC, then HLA haplotype sharing byọ RA sibs should be greater than random and not affected by either DR status of the proband or byo the presence or absence of autoimmune thyroido disease in the sibship.

\section{Patients and methods}

Thirty-five Caucasoid sibships with two or more sibs $\supset$ affected by classical or definite rheumatoid arthritis were studied. ${ }^{4}$ There were 29 pairs, five trios, and one quartet of affected sibs. In two families sib pairs in two generations were considered. Clinical disease $O$ features, the presence or absence of other autoim- $c$ mune diseases, and serum autoantibodies were? documented in all patients. Autoantibodies testedo for included IgM rheumatoid factor (sheep cell, agglutination test (SCAT) by RAHA kit, Fujizoki $\stackrel{\oplus}{+}$ Inc., Tokyo), antithyroglobulin antibodies by Thymune-T (Wellcome), and antithyroid microsomalo antibodies by Thymune-M (Wellcome). The follow- $\Omega$ 
ing titres are found in less than $5 \%$ of a local normal population and were considered positive for each test: SCAT 1/32, antithyroglobulin $1 / 40$, antimicrosomal 1/1600. Sibships which included members (either affected or non-affected by RA) either with histories of definite autoimmune thyroid disease (Hashimoto's disease, primary hypothyroidism with circulating antithyroid autoantibodies, Graves' disease) or who had significant titres of thyroid autoantibodies on serological testing were categorised as 'thyroid sibships' (RA-TH). Haplotype sharing by RA sibling pairs was compared in the thyroid (RA-TH) and non-thyroid (RA-non-TH) sibships.

\section{TISS UE TYPING}

HLA-A and $B$ antigens were defined by the standard NIH technique. HLA-DR antigens were defined with $B$ lymphocytes isolated from peripheral blood by their adherence to nylon wool columns. ${ }^{5}$ All antisera were obtained locally and were characterised using a cell panel typed with seventh, eighth, and ninth International Histocompatibility antisera.

\section{STATISTICAL ANALYSIS}

If HLA haplotypes are inherited at random in a particular sibship the probabilities of any sib sharing 0,1 , or 2 haplotypes with a proband are $0 \cdot 25,0 \cdot 5$, and 0.25 respectively. The probabilities that the observed frequencies of haplotype sharing differ significantly from the random distribution were analysed by assessing the number of times parental haplotypes were repeated in affected siblings. ${ }^{6} \mathrm{p}$ Values of less than $5 \%$ were considered statistically significant.

\section{Results}

Fifteen sibships contained individuals with clinical or immunological evidence of autoimmune thyroid disease. There were no overall clinical differences in rheumatoid disease severity between members of these 'thyroid' sibships and affected members of the other 'non-thyroid' sibships. (82\% of RA subjects in the 'thyroid' and $84 \%$ in the 'non-thyroid' sibships had received 'second-line' drug therapy, and $71 \%$ of RA subjects in 'thyroid' and $65 \%$ in 'non-thyroid' sibships had irreversible articular deformities). $69 \%$ of RA members in the 'thyroid' families and $63 \%$ in the 'non-thyroid' families were seropositive for rheumatoid factor at the time of assessment. Ten of $15(67 \%)$ probands in the thyroid families and 18 of $20(90 \%)$ in the non-thyroid familiees were HLADR4 positive.

HLA haplotype sharing by RA sibs overall was significantly greater than random (Table 1 ). The
Table 1 The relative frequencies of $R A$ sib pairs sharing 0,1 or 2 haplotypes. (No of pairs in parentheses)

\begin{tabular}{llll}
\hline Haplotypes shared & 0 & $l$ & 2 \\
\hline RA overall (42)§ & $0 \cdot 12$ & 0.48 & $0.40^{*}$ \\
& $(5)$ & $(20)$ & $(17)$ \\
RA-TH (19) & $0 \cdot 21$ & 0.47 & 0.32 \\
& $(4)$ & $(9)$ & $(6)$ \\
RA-non-TH (23) & $0 \cdot 04$ & 0.48 & $0.48 \dagger$ \\
DR4 negative proband (8) & $(1)$ & $(11)$ & $(11)$ \\
Random distribution & $0 \cdot 25$ & 0.38 & 0.38 \\
& $(2)$ & $(3)$ & $(3)$ \\
& 0.25 & 0.5 & 0.25 \\
\hline
\end{tabular}

RA-TH=RA sibs in sibships with clinical or laboratory features of autoimmune thyroid disease; RA-non-TH=RA sibs after exclusion of sibships with clinical or laboratory evidence of autoimmune thyroid disease.

${ }^{*} \mathbf{p}=\mathbf{0 . 0 3}$.

$\dagger \mathrm{p}=\mathbf{0 . 0 0 6}$ versus random distribution.

$\S$ Twenty-nine pairs, five trios, and one quartet, i.e. $29+10+3$.

frequencies of HLA haplotype sharing by RA sibs in the 'thyroid sibships' $(p=0.28)$ or where the proband was HLA-DR4 negative were not significantly different from the random assuribution (Table 1). In both instances where a DR4 negative proband had no haplotypes in common with an affected sibling, that sibling was DR4 positive. There was a trend for greater than random haplotype sharing by six affected sibling pairs where both were DR4 negative (three share one and three share two haplotypes; $p=0.097)$. HLA haplotype sharing by RA sibs after exclusion of the thyroid sibships was highly statistically significant $(p=0.006)$.

\section{Discussion}

If a single susceptibility gene within the MHC predisposed to both DR4 positive and negative RA, we would expect to find an equivalent and greater than random HLA haplotype sharing by all the categories of RA sibs examined, irrespective of whether the proband was DR4 positive, negative, or belonged to a 'thyroid' sibship. The near random haplotype sharing by RA sibs in the latter two categories of sibship as compared with the significantly increased haplotype sharing by RA siblings in the 'non-thyroid' sibships suggests that the model of a single RA susceptibility gene linked to the DR locus is incorrect. It seems unlikely that clinical heterogeneity can explain these findings, as overall RA disease severity and frequencies of seropositivity for IgM rheumatoid factor are similar in the 'thyroid' and 'non-thyroid' sibships. An alternative explanation is that another gene (or genes), not in linkage disequilibrium with HLA-DR4, may also predispose to RA in some DR4 negative or 'thyroid disease' 
families. In these families RA may result from either the gene in linkage disequilibrium with DR4 or the independent gene(s), so that increased HLA haplotype sharing by affected sibs need not occur. We cannot say for certain from the present data whether the genetic effect independent of DR4 is within or outside the MHC. However, although the numbers are small, there is a trend for greater than random haplotype sharing by RA siblings where both or all are DR4 negative, suggesting that the former possibility is more likely.

If there is a second susceptibility gene for RA, we should consider whether this is in linkage disequilibrium with any other DR antigen. An immunogenetic subset of RA has been described which is characterised by circulating antibodies to native type II collagen and which is associated with DR3 and $7 .^{7}$ This subset is unlikely to account for the present findings, as patients in the subset do not usually give a family history of RA and most of the patients tested here do not have antibodies to type II collagen. An association between RA and HLADR1 has been described in Israeli $\mathrm{Jews}^{8}$ and in Indian Asians studied in the $\mathrm{UK}^{9}$ but not in a subsequent study of RA in India. ${ }^{10}$ HLA-DR3 may act as a marker for high immune responder genes, as suggested by the association between this antigen and high autoantibody titres, immunological side effects of gold and penicillamine, and certain extraarticular disease features of RA. ${ }^{11}$ We have compared DR antigen frequencies found in a random group of unrelated, DR4 negative, Caucasoid, RA patients seen in Greater Manchester with antigen frequencies found in DR4 negative controls (Table 2). Although there is a slight increase in both DR3 and DR1 in the RA group, neither difference is statistically significant. However, there is a

Table 2 Comparison of $D R$ antigen frequencies in $D R 4$ negative $R A^{*}$ patients with those in DR4 negative controls. (Percentages in parentheses)

\begin{tabular}{lccc}
\hline & $R A(n=79)$ & Controls $(n=261)$ & $p_{c}{ }^{\prime}$ \\
\hline DR1 & $28(35 \cdot 4)$ & $68(26 \cdot 1)$ & 0.71 \\
DR2 & $16(20 \cdot 2)$ & $88(33 \cdot 7)$ & $0 \cdot 15$ \\
DR3 & $36(45 \cdot 6)$ & $90(34 \cdot 5)$ & 0.42 \\
DR5 & $9(11 \cdot 4)$ & $35(13 \cdot 4)$ & \\
DR6 & $12(15 \cdot 2)$ & $61(23.4)$ & 0.79 \\
DR7 & $17(21 \cdot 6)$ & $79(30 \cdot 3)$ & 0.095 \\
DR8 & $1(1.2)$ & $12(4 \cdot 6)$ & \\
DR9 & 0 & $4(1.5)$ & \\
DR10 & 0 & $2(0.7)$ & \\
Blank & $39(49 \cdot 4)$ & $83(31 \cdot 8)$ & 0.035 \\
DR1+3 & $58(73.4)$ & $147(56 \cdot 3)$ & 0.028 \\
\hline
\end{tabular}

*Unrelated British Caucasoids.

$+p_{c}=$ probability corrected for the number of comparisons made (Fisher's test). statistically significant increase in the combined $\stackrel{5}{3}$ frequencies of DR1 and DR3 in the RA group, 드. which suggests that the gene independent of DR 4 ? may be in linkage disequilibrium with both DR1 and $\stackrel{\overrightarrow{\mathrm{F}}}{\vec{*}}$ 3. The increase in DR blanks in the RA groupo (patients typing for one DR antigen only) could suggest either an increased risk for homozygosity in $\overline{\bar{s}}$ DR4 negative RA or more probably a role for a $\mathrm{DR}$ antigen not yet serologically defined.

The above results suggesting genetic heterogeneity in the pathogenesis of RA are of interest in the $\vec{\circ}$ light of recent reports that the frequency of the G1m $\overrightarrow{-}$ $(\times)$ allotype $(\mathrm{Gm}$ is coded for by genes on chromo- $⿴$ some $14^{12}$ ) is increased in DR4 positive but not DR4 negative RA. ${ }^{13} 14$ Thus the two or more genes within the MHC may interact with different non-or HLA linked genes. The second gene within the MHC may also predispose to autoimmune thyroid disease and probably to other autoimmune diseaseso which occur in relatives of RA probands. Autoimmune thyroid disease may be less frequent in relatives of patients with sporadic RA than in ouro multicase families. ${ }^{15}$

Comparison of our observed frequencies for HLA haplotype sharing by affected sibs in 'non-thyroid' $\overrightarrow{0}$ families with those expected for different freques cies of a dominant or recessive gene linked $\tau_{0}^{\circ}$ $\mathrm{HLA}^{16}$ suggests that the susceptibility gene linkage disequilibrium with HLA-DR4 is dominant and has a frequency of about $5 \%$. The frequencieso of homozygote and heterozygote genotypes can be $\frac{0}{\mathrm{O}}$ calculated from this gene frequency by the Hardy Weinberg formula, and if the prevalence of RA isō $2 \%$, these calculations suggest a gene penetrance of around $20 \%$.

We are grateful to the North West Regional Health Authority and to the Arthritis and Rheumatism Council for financial support. We are indebted to the following colleagues from Greater Manchester. who have referred some of the families studied or allowed us to see? their patients: Dr R Clague, Dr W Dodds. Dr Fallowfield, Dr R윽 Hilton, Professor M I V Jayson, Dr J Marks, Dr J Sharp, Dr P Smith, and Dr D Swinson. The autoantibody tests were carried outo by courtesy of $\mathrm{Dr} M$ Haeney, Subregional Department of Immunology, Hope Hospital, Salford.

\section{References}

1 Lawrence I S: Rheumatoid arthritis In: Rheumatism in $N$ populations. London: Heinemann, 1977: 156-271.

2 Stastny P. Rheumatoid arthritis. In: Terasaki P I, ed. Histocom-

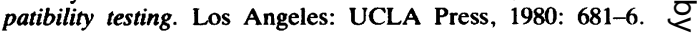

3 Grennan D M, Dyer P A, Clague R, Dodds W, Smeaton I 0 Harris R. Family studies in RA-the importance of HLA-DR4 and of genes for autoimmune thyroid disease. $J$ Rheumatol 1983; 10: 584-9.

4 Ropes M W. Diagnostic criteria for rheumatoid arthritis 1958 국 revision. Ann Rheum Dis 1959; 18: 49-53.

5 Danilovs J A, Ayoub G, Terasaki P I. B lymphocyte isolation 
by thrombin-nylon wool. In: Terasaki P I, ed. Histocompatibility testing. Los Angeles: UCLA Press, 1980: 287-8.

6 Green J R, Woodrow J C. Sibling method for detecting HLAlinked genes in disease. Tissue Antigens 1977; 9: 31-5.

7 Dyer P, Clague R B, Klouda P T, Frith S, Harris R, Holt P J L. HLA antigens in patients with rheumatoid arthritis and antibodies to native type II collagen. Tissue Antigens 1983; 20: 394-6.

8 Schiff B, Mizrachi Y, Orgad S, Yaron M, Gazit E. Association of HLA-Aw31 and HLA-Dr1 with adult rheumatoid arthritis. Ann Rheum Dis 1982; 41: 403-4.

9 Woodrow J C, Nichol F E, Zaphiropoulos G. DR antigens and rheumatoid arthritis: a study of two populations. Br Med J 1981; 283: 1287-8.

10 Mehra N K. Vaidya M C, Taneya V, et al. HLA-DR antigens in rheumatoid arthritis in North India. Tissue Antigens 1982; 20: 300-2.

11 Panayi G S, Wooley P, Batchelor J R. Genetic basis of rheumatoid disease. HLA antigens, disease manifestations and toxic reactions to drugs. $\mathrm{Br}$ Med $J$ 1978; ii: 1326-8.

12 Cox D W, Markovic V D, Teskima I E. Genes for immunoglobulin heavy chain and for alpha-1-antitrypsin are localised to specific regions of chromosome 14q. Nature 1982; 297: 428-30.

13 Propert D N, Kay P, McCluskey J, Zilko P J, Mathews J. Immunoglobulin allotypes in rheumatoid arthritis. In: Immunogenetics of rheumatoid arthritis. Amsterdam: Excerpta Medica, 1983; 313: 127-9.

14 Sanders P A, De Lange G G, Dyer P A, Grennan D M. Gm and $\mathrm{Km}$ allotypes in rheumatoid arthritis. Ann Rheum Dis 1985; 44: 529-32.

15 Walton K, Dyer P A. Grennan D M, Haeney M, Harris R. Clinical features, autoantibodies and HLA-DR antigens in rheumatoid arthritis. J Rheumatol 1985; 12: 223-6.

16 Thomson G, Bodmer W. The genetic analysis of HLA and disease associations. In: HLA and disease. Copenhagen: Munksgaard, 1977: 84-93. 